\title{
Is tapping jet nebulisers worthwhile?
}

\author{
Mark L Everard, Margaret Evans, Anthony D Milner
}

\begin{abstract}
An in vitro system was used to study the effect of tapping a jet nebuliser on the dose delivered to patients. Using a $2 \mathrm{ml}$ fill, effective drug delivery had ceased by 3 minutes but a further $38 \%$ was delivered to the filter by 5 minutes if the chamber was tapped.
\end{abstract}

(Arch Dis Child 1994; 70: 538-539)

Determining when to turn a jet nebuliser off has always been a very subjective decision. It has variously been suggested that nebulisation be discontinued when the nebuliser output becomes intermittent, when the output appears to cease, when the output appears to cease completely while tapping the nebuliser, when the 'nebuliser is dry', 1 or when an arbitrary time limit has been reached. ${ }^{2}$ There is, however, very little information available on which to base rational advice for clinical purposes. For this reason, a previously described method ${ }^{3}$ was used to determine what effect altering the criteria used to terminate a period of treatment might have on the dose delivered to a patient from a jet nebuliser.

\section{Method}

The in vitro model used a Starling ventilator to simulate the respiratory pattern of an individual. This model allows us to determine the effect of using a variety of operating conditions on the dose inhaled from a jet nebuliser while maintaining a constant respiratory pattern. A tidal volume of $50 \mathrm{ml}$ and a respiratory rate of 32 breaths per minute were chosen as these were used in the previous study. The dose of sodium cromoglycate 'inhaled' from a Cirrus jet nebuliser was collected on a filter and the dose assayed using a spectrophotometric assay as previously described. ${ }^{3}$ A driving gas flow of $81 / \mathrm{min}$ was used. A $2 \mathrm{ml}$ fill of $1 \%$ sodium cromoglycate was used for three experiments in which the solutions were nebulised until nebulisation became intermittent $(1.5 \mathrm{~min})$, appeared to cease $(3 \mathrm{~min})$, a total of 5 minutes and for 5 minutes with the nebuliser chamber being tapped from the point at which nebulisation became intermittent. A fifth experiment was performed in which $4 \mathrm{ml}$ of a $1 \%$ solution was nebulised for 5 minutes, the increased volume ensuring that nebulisation was continuous for the whole 5 minute period. Each experiment was repeated four times.

The osmolality of the sodium cromoglycate solution was measured before and after 5 minutes of nebulisation using a standard osmometer for both a $4 \mathrm{ml}$ fill and a $2 \mathrm{ml}$ fill with the chamber being tapped.
Results

The results from the Starling pump experiments are presented in the figure. When $4 \mathrm{ml}$ of a $1 \%$ solution of sodium cromoglycate were nebulised for 5 minutes the osmolality increased from 40 to $45 \mathrm{mOsm}$ while the osmolality of $2 \mathrm{ml}$ of a $1 \%$ solution increased from 40 to $54 \mathrm{mOsm}$ in a 5 minute period when the chamber was being tapped.

\section{Discussion}

These results show that when using a conventional jet nebuliser at a constant driving gas flow, the dose delivered to a patient in a given period can be greatly influenced by altering the criteria used to define when a period of nebulisation has been completed. Drug delivery continued after output became intermittent but effective nebulisation had ceased by 3 minutes with no further drug being delivered when nebulisation was continued to 5 minutes. The time to the end of effective nebulisation will be longer if lower driving flows are used. Tapping the nebuliser from the point at which nebulisation became intermittent resulted in a $38 \%$ increase in the dose delivered to the filter compared with that when the nebuliser was held still.

Tapping a nebuliser causes droplets adhering to the internal walls to fall to the base of the chamber and hence they become available for nebulisation. In these experiments, tapping extended the period of continuous nebulisation but output still became intermittent, and indeed appeared to cease, by 5 minutes. Hence it was a little surprising that the dose deposited on the filter, when tapping, approached that delivered when a $4 \mathrm{ml}$ fill was nebulised for 5 minutes. It is probable that the more rapid increase in solution concentration that occurs using a $2 \mathrm{ml}$ rather than a $4 \mathrm{ml}$ fill contributes to this result. The concentration of drug within an aerosol generated from a

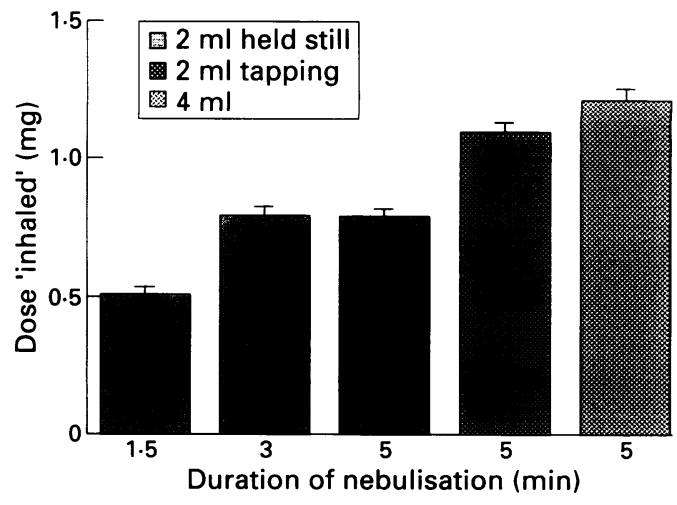

Effect of altering criteria for ceasing nebulisation on dose delivered when using a $2 \mathrm{ml}$ fill compared with dose delivered from 5 minutes continuous nebulisation of a $4 \mathrm{ml}$ fill. 
solution reflects the solution concentration and it has previously been shown that aerosol concentration is a major determinant of the rate of drug delivery. ${ }^{3}$

In these experiments; air entrainment is unlikely to be significant because of the small tidal volume used. Our previous study ${ }^{3}$ would predict that the absolute dose delivered to the filter may vary with tidal volume but the observed trends are not likely to be significantly affected.

For $\beta_{2}$ sympathomimetics, the extra drug delivery resulting from tapping a nebuliser is unlikely to be significant since supramaximal doses are used while tapping increases the treatment times. For other drugs, such as antibiotics, this extra dose may be valuable. It is likely that for many drugs, the convenience of other delivery systems such as metered dose inhalers with holding chambers, ${ }^{4}$ novel systems for delivering wet aerosols, and effort independent powder delivery systems will mean that jet nebulisers will gradually become obsolete. In the meantime, the convenience and acceptability of this form of treatment would be improved by attempting to shorten rather than extend treatment times. ${ }^{3}$ The most effective way would be to use more concentrated solutions, use smaller fill volumes, and design nebulisers with smaller 'dead volumes' rather than rely on measures such as diluting solution and tapping the nebuliser chamber.

Dr Everard was generously supported by the National Asthma Campaign.

1 Zainudin BMZ, Biddiscombe $M$, Tolfree SEJ, Short $M$, Spiro SG. Comparison of bronchodilator responses and deposition patterns of salbutamol inhaled from a pressurised metered dose inhaler, as a dry powder, and as a nebulised solution. Thorax 1990; 45: 469-73.

2 O'Callaghan C, Clark AR, Milner AD. Why nebulise for more than five minutes? Arch Dis Child 1989; 64: 1270-3. 3 Everard ML, Clark AR, Milner AD. Drug delivery from jet nebulisers. Arch Dis Child 1993; 67: 580-5.

4 Newhouse MT. Emergency department management of lifethreatening asthma: are nebulizers obsolete? [Editorial.] Chest 1993; 103: 661-3. 